\title{
Simulation des Mikrorisswachstums bei zyklischer Beanspruchung
}

\author{
Zenner, Harald
}

\author{
Veröffentlicht in: \\ Abhandlungen der Braunschweigischen \\ Wissenschaftlichen Gesellschaft Band 52, 2002, \\ S.147-156
}

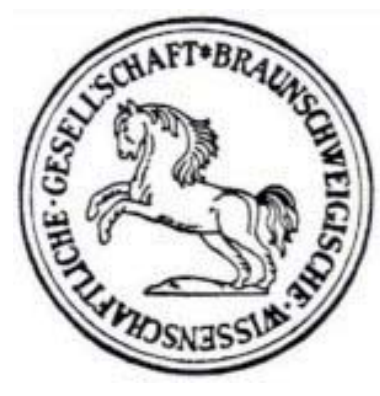

J. Cramer Verlag, Braunschweig 


\title{
Simulation des Mikrorisswachstums bei zyklischer Beanspruchung
}

\author{
von Harald Zenner, Clausthal-Zellerfeld*
}

(Eingegangen am 20.03.2002)

\section{Einführung}

Die Treffsicherheit der Lebensdauerberechnung zyklisch beanspruchter Bauteile ist nach wie vor unbefriedigend [1]. Dies liegt einmal daran, dass die Vorgänge, die zur Mikrorissentstehung, zum Mikrorisswachstum und schließlich zum makroskopischen Rissfortschritt und Bruch führen, äußerst komplex sind, zum anderen sind die Schädigungsmechanismen, die sich im Werkstoff abspielen und zum Versagen eines Bauteils führen können, nicht vollständig verstanden. Die heute verwendeten Berechnungskonzepte behandeln die Schadensakkumulation rein phänomenologisch (Nennspannungskonzept, Örtliches Konzept), d.h. ohne Berücksichtigung mikrostruktureller Vorgänge.

In den letzten Jahren ist das Verständnis des Mikrorisswachstums erweitert worden $[2,3]$. Das Risswachstum in duktilen metallischen Werkstoffen verläuft zunächst in stage I (microstructural short cracks) innerhalb des Korns, getrieben durch die Schubspannung. Mit Annäherung an eine Barriere, z.B. Korngrenze, verlangsamt sich das Risswachstum. Nach Erreichen einer spezifischen Risslänge (mehrere Körner) erfolgt das Wachstum in stage II, d.h. als Normalspannungsriss (physical small crack).

Damit bietet es sich an, das Mikrorisswachstum zu modellieren und zu simulieren und mit experimentellen Befunden zu vergleichen mit dem Ziel, die Schädigungsmechanismen besser zu verstehen und damit die Schadensakkumulation in der Lebensdauerberechnung realistischer als bisher beschreiben zu können. Die hier betrachtete Lebensdauerphase von der Risskeimbildung bis zum makroskopischen Anriss kann je nach Geometrie, Beanspruchungshöhe und Werkstoff dominierend für ein Bauteil sein.

\section{Modellbildung und Simulation}

Im Simulationsmodell wird die Oberfläche eines polykristallinen Werkstoffs durch zweidimensionale hexagonale Elemente abgebildet, Bild 1. Im Gegensatz zum Gleitebenensystem eines realen Kristalls wird hier jedem Korn nur eine Gleitebenenrichtung zugeordnet, die zufällig ist. Die Spannungen in der Gleitebenenrichtung werden in Abhängigkeit von den äußeren Spannungen berechnet. Zu Beginn der Simulation werden bei vorgegebener Risskeimdichte punktförmige Risskeime erzeugt, deren Position durch eine

* Prof. Dr.-Ing Harald Zenner · Siebensternweg 22 - D-38678 Clausthal-Zellerfeld

Kurzfassung eines Vortrags vor der Klasse für Ingenieurwissenschaften der Braunschweigischen Wissenschaftlichen Gesellschaft am 09.02.2002 in Braunschweig. 


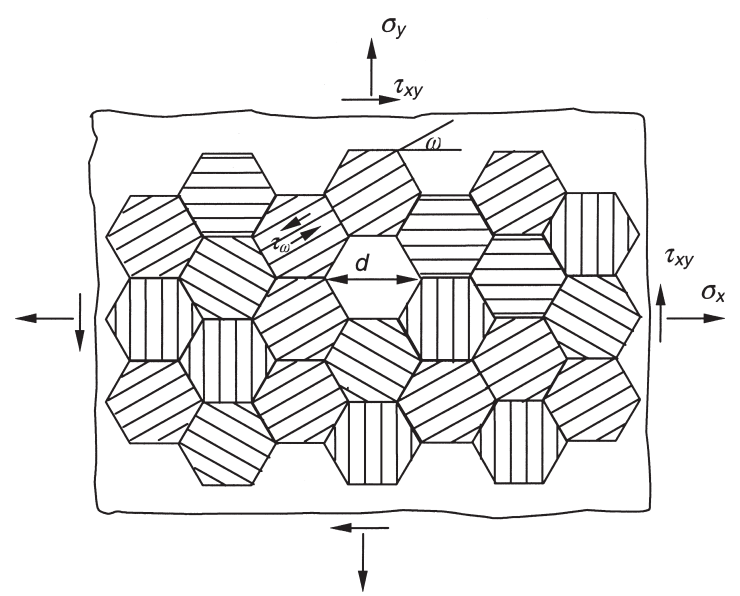

Bild 1: Modellierung der Mikrostruktur und Spannungszustand

Zufallsfunktion bestimmt wird. Die Risswachstumsrate in stage I wird unter Verwendung von Gl. (1) simuliert:

$$
\left(\frac{\mathrm{da}}{\mathrm{dN}}\right)_{\omega}=\mathrm{A}\left(\Delta \tau_{\omega}\right)^{\alpha} \cdot(\mathrm{d}-\mathrm{a})
$$

wobei

$$
\left(\frac{\mathrm{da}}{\mathrm{dN}}\right)_{\omega} \text { - Risswachstumsrate in der Ebene } \omega
$$

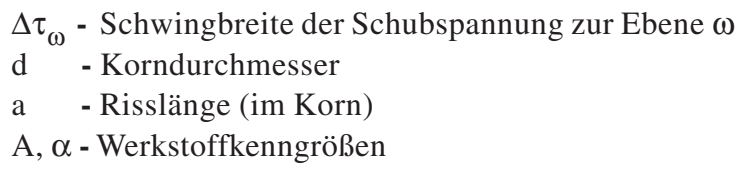

Gl. (1) sagt aus, dass die Risswachstumsrate mit zunehmender Risslänge abnimmt. Erreicht die Risslänge den Korndurchmesser, d.h. wird der Abstand der Rissspitze zur Barriere null, so wird auch die Risswachstumsrate null.

Risswachstum kann konkurrierend auch durch Normalspannungen hervorgerufen werden, Gl. (2):

$$
\left(\frac{\mathrm{da}}{\mathrm{dN}}\right)_{\omega}=\mathrm{B}\left(\Delta \sigma_{\omega}\right){ }^{\beta} \mathrm{a}^{\chi}-\mathrm{C}
$$

wobei

$$
\begin{aligned}
& \Delta \sigma_{\omega} \text {-Schwingbreite der Normalspannung zur Ebene } \omega \\
& \mathrm{B}, \mathrm{C}, \beta, \chi \text { - Werkstoffkenngrößen }
\end{aligned}
$$


Weitere Ansätze berücksichtigen das Sichverbinden (Koaleszenz) benachbarter Mikrorisse sowie die Rissumlenkung von stage I zu stage II, d.h. vom Schubspannungs- zum Normalspannungsriss.

Das Flussdiagramm ist in Bild 2 dargestellt. Die Lebensdauerberechnung erfolgt bis zu einer makroskopischen Anrisslänge von $500 \mu \mathrm{m}$.

Es sei darauf hingewiesen, dass das vorliegende Modell bewusst Vereinfachungen enthält, vergl. [4], einmal um zunächst die Aussagefähigkeit eines solchen Modells zu überprüfen, aber auch um die Schwierigkeiten bei der Parameteridentifikation klein zu halten.

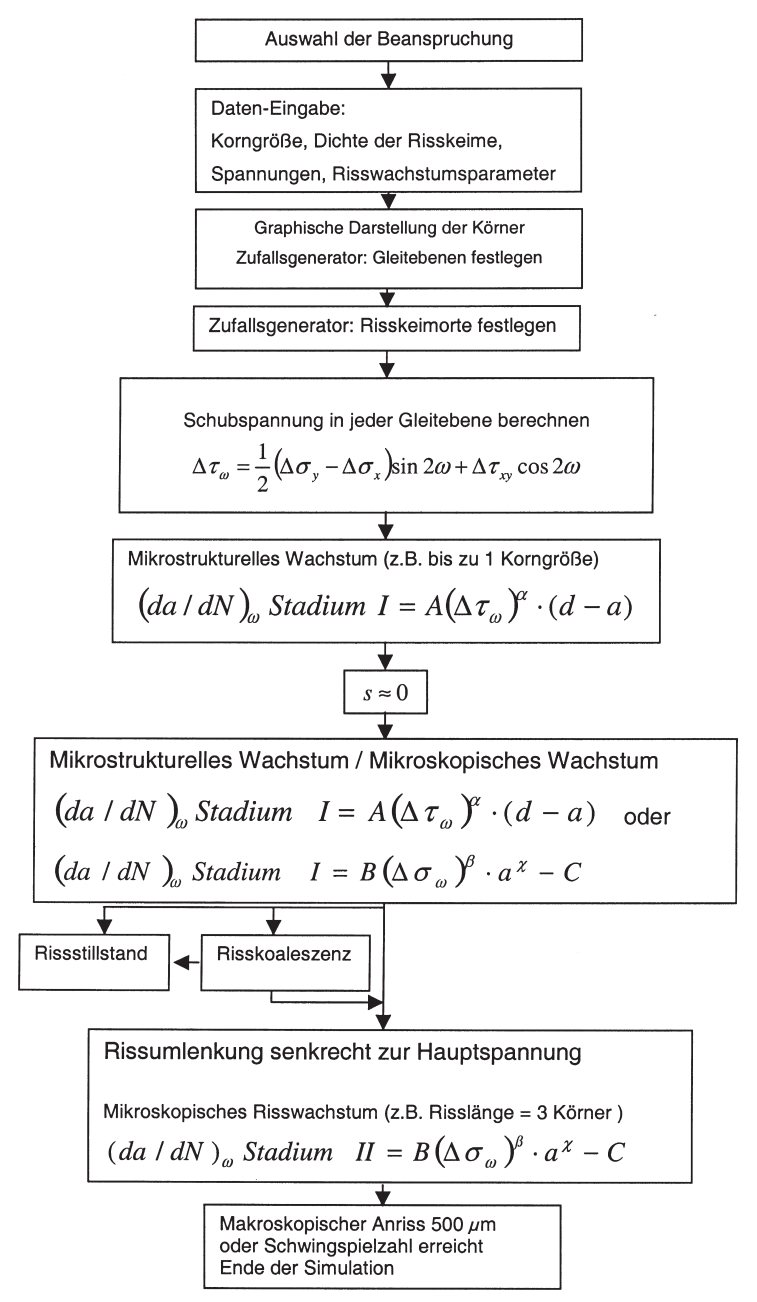

Bild 2: Flussdiagramm der Simulation 


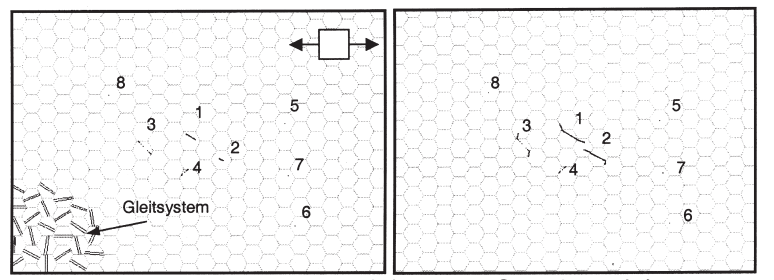

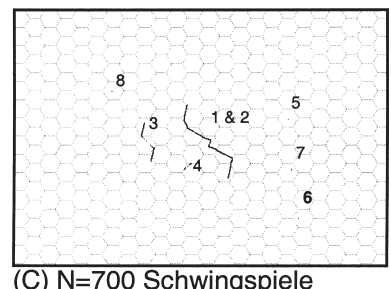

(C) $N=700$ Schwingspiele

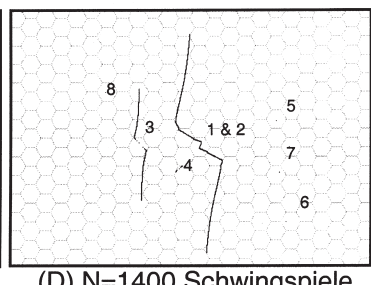

(D) $\mathrm{N}=1400$ Schwingspiele

Bild 3: Risssimulation für Zug-Druck

\section{Simulationsbeispiele}

Bild 3 zeigt das simulierte Mikrorisswachstum unter Zug-Druck-Beanspruchung (die zufällige Orientierung der Gleitebenen ist auf Bild (A) unten links beispielhaft gezeigt). Dargestellt ist der Mikrorissverlauf für unterschiedliche Schwingspielzahlen. Die punktförmigen Risskeime wachsen zunächst in Richtung der Gleitebene des jeweiligen Korns, getrieben durch die in der Gleitebene wirkende Schubspannung (Riss 1 und 2, Bild A und B). Vorzugsweise wachsen die Mikrorisse bei Zug-Druck-Beanspruchung unter $\omega$ $= \pm 45^{\circ}$, weil dort die Schubspannungen am größten sind. Bei einer Annäherung der Rissspitzen zweier Mikrorisse erfolgt Koaleszenz (Riss 1 und 2, Bild C). Bei Erreichen einer kritischen Risslänge findet der Übergang von stage I zu stage II statt (Bild C und D). Schließlich ist der makroskopische Anriss erreicht.

Bild 4 zeigt die Risslänge über der Schwingspielzahl für die Risse in Bild 3. Zunächst wächst der Riss innerhalb des Korns relativ schnell. Mit Annäherung an die Korngrenze, hier $60 \mu \mathrm{m}$, nimmt die Risswachstumsrate ab. Einzelne Risse können stehen bleiben. Erst wenn die Risslänge den Korndurchmesser überschreitet, nimmt die Risswachstumsrate deutlich zu, wobei durch Risskoaleszenz eine sprunghafte Zunahme auftritt. Im weiteren Verlauf wird die makroskopische Risslänge von $\mathrm{a}=500 \mu \mathrm{m}$ schnell erreicht.

Wird die jeweils größte Risslänge als Maß für die Schädigung angenommen, Bild 4, so ist zu erkennen, dass die Zunahme der Schädigung nicht linear und, bedingt durch die Risskoaleszenz, nicht kontinuierlich erfolgt. Der zu einem bestimmten Zeitpunkt auftretende größte Riss muss nicht zum makroskopischen Anriss führen. Die Lebensdauer wird 


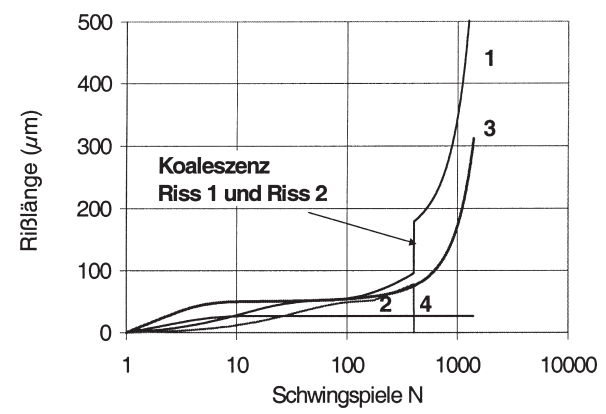

Bild 4: Risslänge über der Schwingspielzahl für Zug-Druck (Risse aus Bild 3)

ganz wesentlich durch die Dauer der mittleren Phase bestimmt. Diese Phase ist dadurch geprägt, dass sich der Mikroriss der Barriere (hier Korngrenze) nähert und die Risswachstumsrate sehr niedrige Werte annimmt.

Der Einfluss der Korngröße auf das Mikrorisswachstum wurde für d = 45, 60 und 120 $\mu \mathrm{m}$ bei einer Risskeimdichte von $30 \mathrm{~mm}^{-2}$ untersucht. Wie Bild 5 zeigt, nimmt die ertragbare Schwingspielzahl bis zum makroskopischen Anriss mit zunehmender Korngröße ab. Auch hier ist zu erkennen, dass der Riss zunächst innerhalb des Korns schnell wächst. Mit Annäherung des Risses an die Barriere (Korngrenze) tritt eine Phase langsamen Risswachstums ein, Bild 5a und 5b. Diese Phase verkürzt sich mit zunehmender Korngröße, da der „Ausgangsriss“ für diese Phase mit zunehmender Korngröße zunimmt. Für $\mathrm{d}=120 \mu \mathrm{m}$ geht die Phase langsamen Risswachstums praktisch verloren, Bild 5c.

\section{Vergleich Simulation und Experiment}

Die Zunahme der Risswachstumsrate mit zunehmender Korngröße ist aus vielen experimentellen Arbeiten bekannt, vergl. z.B. [5].

Für den Vergleich zwischen Simulation und Experiment ist der sog. Zweistufenversuch interessant. Bekannt ist, dass für eine High-Low-Lastfolge die Lebensdauer geringer ist als für eine Low-High-Lastfolge, wie das zum Beispiel Versuche in [6] zeigen. Wie aus Bild 6 zu entnehmen ist, müssten nach der linearen Schadensakkumulation von PalmgrenMiner die Versuchspunkte auf der Geraden zwischen $\mathrm{D}_{1}+\mathrm{D}_{2}=1$ liegen. Für Low-High (LH) liegen aber alle Versuchspunkte über dieser Geraden und für High-Low (HL) fast alle darunter. Dies lässt sich erklären, wenn man die Risswachstumskurven für beide Laststufen und die Reihenfolge der Belastungen betrachtet.

Eingangsgrößen der Simulation sind d $=60 \mu \mathrm{m}$ und die Risskeimdichte $30 \mathrm{~mm}^{-2}$. Die beiden Spannungshorizonte sind $\sigma_{\mathrm{a} \text { High }}=330 \mathrm{MPa}$ und $\sigma_{\mathrm{aLow}}=254 \mathrm{MPa}$.

Durch Simulation werden für beide Horizonte die Risswachstumskurven a $(\mathrm{N})$ bestimmt, Bild 7. Wird bei High-Low auf dem oberen Horizont begonnen, dann erreicht er z.B. nach $\mathrm{n}_{1}=940$ Schwingspielen eine Länge von $\mathrm{a}_{1}=45 \mu \mathrm{m}$. Die Schwingspielzahl bis zum 


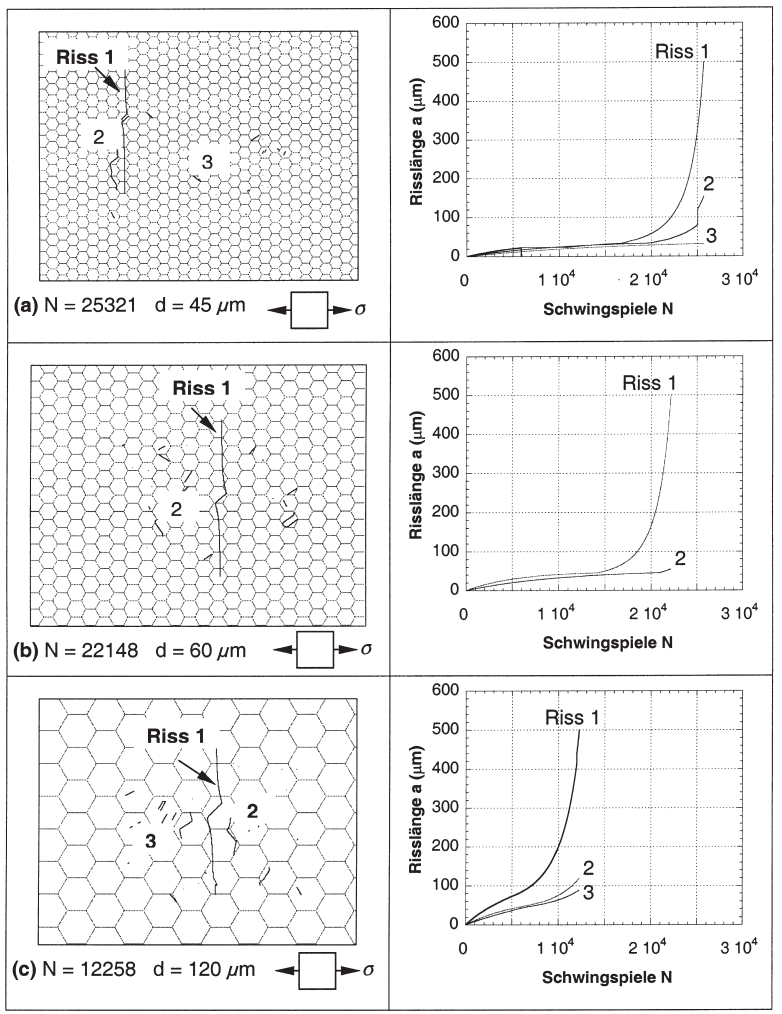

Bild: 5: Risswaschstum bei verschiedenen Korngrößen $\sigma=254 \mathrm{MPa}$, Zug-Druck

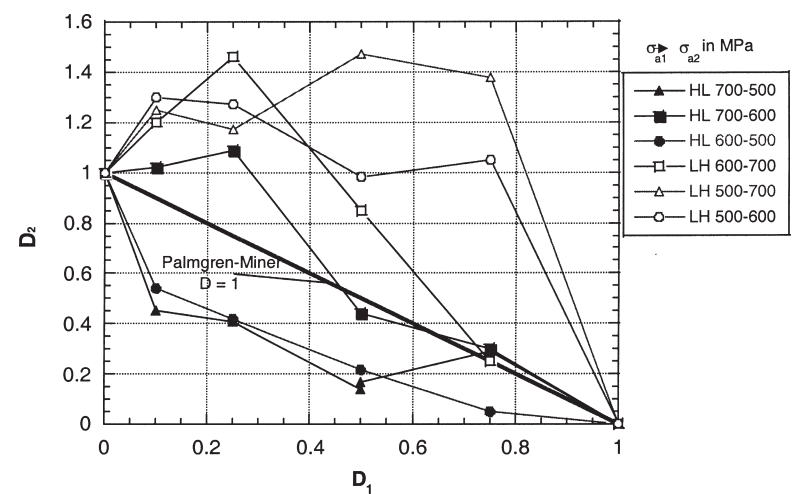

Bild 6: Schadensakkumulation bei Zweistufenbeanspruchung High-Low und Low-High im Experiment, Stahl 42CrMo4, nach [6]. 


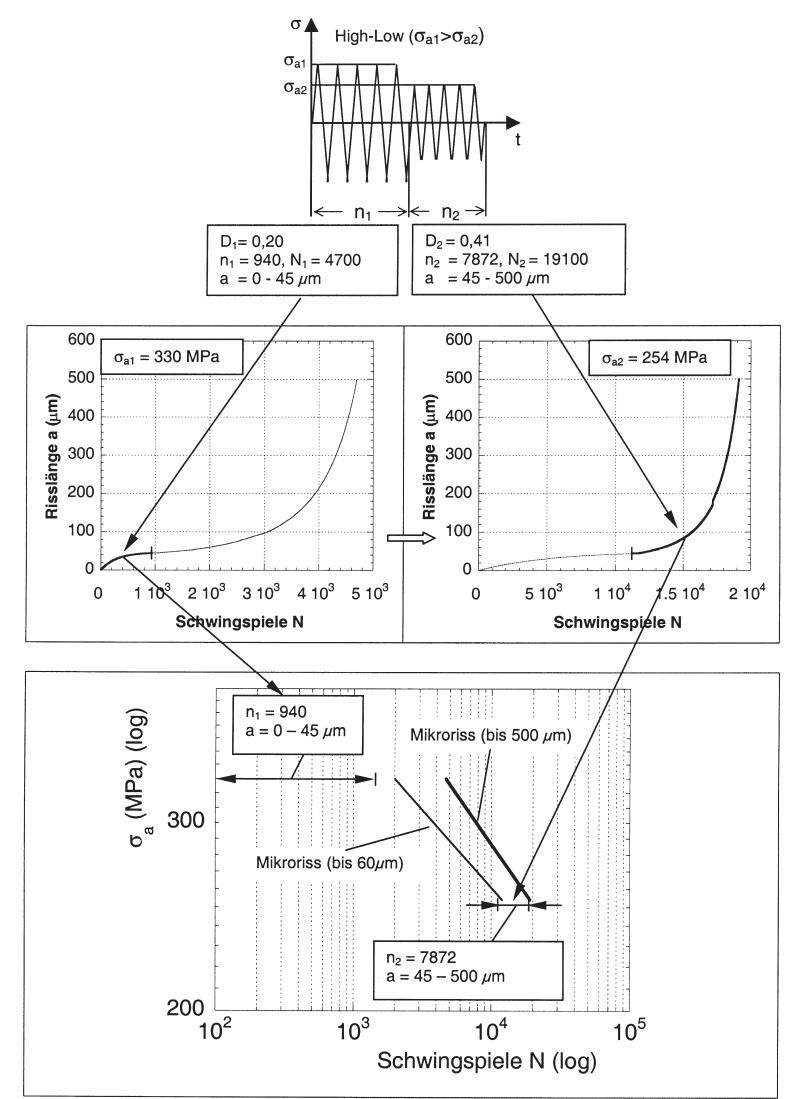

Bild 7: Rissausbreitung und Schadensakkumulation für eine High-Low-Lastfolge

makroskopischen Anriss (500 $\mu \mathrm{m})$ würde bei $\mathrm{N}_{1}=4700$ liegen. Die Schadenssumme nach der linearen Schadensakkumulationshypothese beträgt damit

$$
\mathrm{D}_{1}=\frac{\mathrm{n}_{1}}{\mathrm{~N}_{1}}=0,20
$$

Bei Übergang auf den niedrigeren Horizont beginnt das Risswachstum bei der Risslänge $\mathrm{a}_{1}=45 \mu \mathrm{m}$. Bis zum Erreichen des makroskopischen Anrisses von $500 \mu \mathrm{m}$ werden $\mathrm{n}_{2}=7872$ Schwingspiele benötigt. Bei einer Schwingspielzahl allein auf dem unteren Horizont bis zum makroskopischen Anriss von $\mathrm{N}_{2}=19100$ bedeutet das eine Schadenssumme von

$$
\mathrm{D}_{2}=\frac{\mathrm{n}_{2}}{\mathrm{~N}_{2}}=0,41
$$


Die Gesamtschadenssumme beträgt damit

$$
\mathrm{D}_{\text {High-Low }}=\mathrm{D}_{1}+\mathrm{D}_{2}=0,61
$$

Für eine Low-High-Lastfolge kann auf denselben Risswachstumskurven analog vorgegangen werden, Bild 8. Auf dem unteren Horizont ergibt sich bis zu einer Risslänge $a_{1}=26$ $\mu \mathrm{m}$ eine Schadenssumme

$$
\mathrm{D}_{1}=\frac{\mathrm{n}_{1}}{\mathrm{~N}_{1}}=\frac{3820}{19100}=0,20
$$

Auf dem oberen Horizont beträgt die Schadenssumme

$$
\mathrm{D}_{2}=\frac{\mathrm{n}_{2}}{\mathrm{~N}_{2}}=\frac{4462}{4700}=0,95
$$

Die Gesamtschadenssumme wird dann

$$
\mathrm{D}_{\text {Low-High }}=\mathrm{D}_{1}+\mathrm{D}_{2}=1,15
$$

Aus der Simulation ergibt sich damit für eine

und eine

$$
\text { High-Low-Lastfolge D }<1
$$

$$
\text { Low-High-Lastfolge D > } 1
$$

Der Grund lässt sich unmittelbar aus den Diagrammen ablesen. Der für die Lebensdauer wichtigste mittlere Anteil des Risswachstums, d.h. der Anteil mit der niedrigsten Risswachstumsrate, kann bei High-Low auf beiden Horizonten übersprungen werden, Bild 7. Bei Low-High wird er mindestens einmal auftreten, Bild 8. Das Gesamtergebnis der Simulation ist in Bild 9 dargestellt und mit der linearen Schadensakkumulation nach Palmgren-Miner verglichen.

Der Vergleich mit Zweistufenversuchen an glatten Probestäben [6] bestätigt weitgehend das Simulationsergebnis, Bild 6. Die relativ große Streuung der Versuchsergebnisse kann möglicherweise darauf zurückgeführt werden, wie Bild 7 und Bild 8 zeigen, dass die sich ergebende Schadenssumme sehr sensibel auf die tatsächlich erreichte Risslänge bei Wechsel des Horizontes reagiert. Im Versuch wurden auch Schadenssummen $\mathrm{D}_{2}>1$ gefunden. Das bedeutet, dass die ertragbare Schwingspielzahl auf dem zweiten Horizont bei einer Vorbelastung auf dem ersten Horizont größer ist als ohne diese Vorbelastung. Ein solches Verhalten kann mit den Mechanismen, die im Simulationsmodell verwendet werden, allerdings nicht beschrieben werden.

\section{Zusammenfassung und Ausblick}

Mit einem einfachen Modell konnte das Mikrorisswachstum vom Risskeim bis zum makroskopischen Anriss unter Schwingbeanspruchung simuliert werden. Dabei bilden sich zunächst Schubspannungsrisse stage I, die in Normalspannungsrisse stage II überge- 


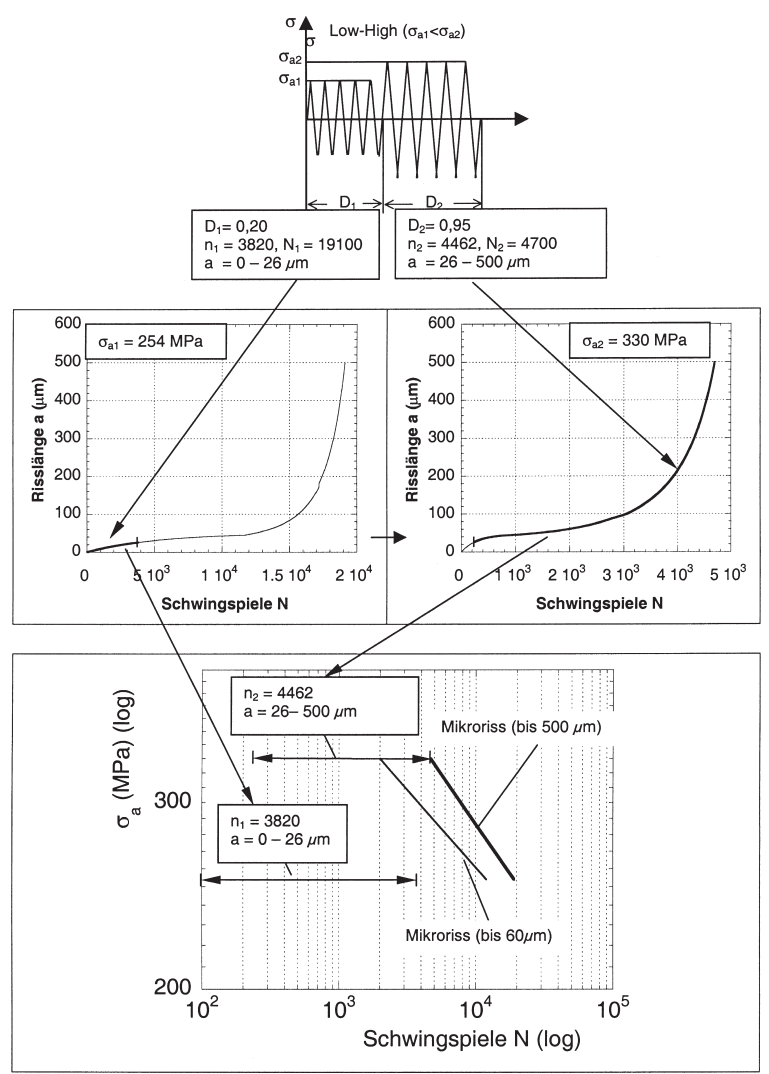

Bild 8: Rissausbreitung und Schadensakkumulation für eine Low-High Lastfolge

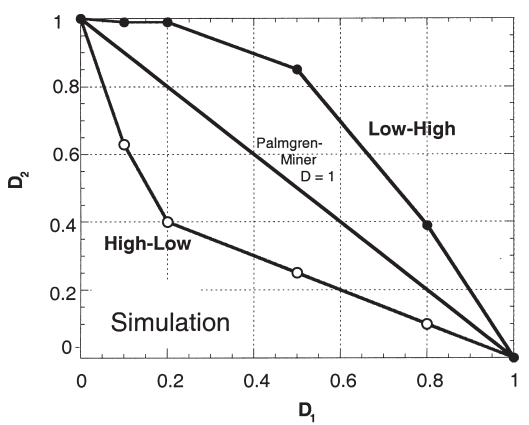

Bild 9: Simulierte Schadensakkumulation bei Zweistufenbeanspruchung High-Low und Low-High 
hen. Das Risswachstum kann in drei Phasen eingeteilt werden. Zunächst erfolgt ein relativ schnelles Risswachstum innerhalb des Korns (Phase I), das sich mit der Annäherung der Rissspitze an die Barriere (Korngrenze) verlangsamt und auch zum Stillstand kommen kann (Phase II). Der Hauptteil der Lebensdauer liegt in dieser Phase. Es folgt eine Phase progressiven Risswachstums bis zum makroskopischen Anriss (Phase III), die vielfach durch Risskoaleszenz eingeleitet wird.

Die Ergebnisse der Simulation zeigen durchweg eine qualitativ gute Übereinstimmung mit experimentellen Ergebnissen. Dies betrifft insbesondere die Ausbreitungsrichtung der Mikrorisse und den Einfluss auf die Lebensdauer. - Hinzuweisen ist jedoch darauf, dass das Modell eine Reihe von Vereinfachungen enthält, die bei einem Vergleich mit Versuchsergebnissen zu beachten sind. So ist z.B. die Korngrenze als Barriere für Mikrorisswachstum nicht für alle metallischen Werkstoffe charakteristisch.

Wird die maximale Mikrorisslänge als Schädigung interpretiert, so erweist sich nach dem vorliegenden Modell die Schadensakkumulation als nicht linear und vielfach als unstetig.

\section{Literatur}

[1] Zenner, H., Lebensdauerkonzepte - Beschreibung, Kritik, Entwicklungen. DVMBerichte 800 „Bauteillebensdauer Nachweiskonzepte“, S. 9-35, 1997.

[2] Miller, K.J. \& E. R. DE Los Rios (eds), The behaviour of short fatigue cracks. EGF (ESIS) Publication No. 1, MEP Institute of Mechanical Engineers, 1986, London, 560 pp.

[3] Miller, K.J., Metal-Fatigue - Past, Current and Future. Proceedings of the Institution of Mechanical Engineers, 1991.

[4] Zenner, H., K. Pötter, A. Schram \& H.A. Suhartono, Simulation des Mikrorisswachstums unter Schwingbeanspruchung, Teil 1: Modell und Simulationsergebnisse, Mikrorisswachstum, Streuung, Einfluss von Beanspruchungsart und -höhe, Risskeimdichte und Korngröße, Teil 2: Simulationsergebnisse - Einfluss des Spannungszustandes und Reihenfolgeeffekte, Mat.-wiss. und Werkstofftech. 32, 33-44 (2001), Mat.-wiss. und Werkstofftech. 33, 845-857 (2002)

[5] Lütjering, G., A. Gysler \& J. Albrecht, Influence of Microstructure on Fatigue Resistance. Proc. Of the Sixth International Fatigue Congress „Fatigue 96“, 1996, Vol. II, pp. 893-904

[6] Schulze, V., K.-H. Lang \& E. Macherauch, Ein- und mehrstufige totaldehnungsund nennspannungskontrollierte Wechselverformungsexperimente an einem Stahl von Typ 42 CrMo 4 unter Eigen- und Mittelbeanspruchungsvariation. Forschungshefte FKM Sonderheft „Bauteilschwingfestigkeit“, 1995, Kapitel 2. 